\title{
Analysis of Network Mass Incident from the Perspective of Audience Standard:Thoughts from "Stranger" Theory
}

\author{
Mingxuan Fan ${ }^{1, a}$ Youqiang $\mathrm{Wei}^{2 b}$ \\ 1.2School of Journalism and Communication, Jiangxi Normal University, China \\ afanmingxuan@outlook.com \\ b190555223@qq.com
}

Keywords: Network mass incident,Audience psychology, We media era,Classification.

\begin{abstract}
In recent years, mass incidents in the offline life have been greatly reduced and gradually faded out of sight. However, due to the deepening of Web3.0 technology and the popularity of the we media, information is more interactive and more targeted for dissemination. Therefore, people in the age of information explosion are more likely to participate in network mass incidents. Since 2006, the research on network group events has been increasing year by year, and it has become a declining trend since 2013. Research has become more and more specialized, but at the same time, it has presented a single perspective, research duplication, etc., so this paper will sort out existing studies. Taking the perspective of the audience as the starting point, this paper analyzes the behavior of network clusters.
\end{abstract}

\section{以受众本位的角度分析网络群体性事件 一一由“陌生人”理论引发的思考 禁明轩 $1, \mathrm{a}$ 魏友强 $2, \mathrm{~b}$ \\ 1.2江西师范大学新闻与传播学院, 南昌, 江西, 中国 afanmingxuan@outlook.com b190555223@qq.com}

关键词：网络群体性事件 受众心理 自媒体时代 分类

中文摘要. 近年来线下生活中的群体性事件大幅减少, 逐渐淡出人们的视野。但是由于 Web3.0 技术的深入、自媒体的普及，信息的交互性更强且传播更具针对性，身处信息爆炸时代的人 们则更易参与到网络群体性事件中。自 2006 年起针对网络群体性事件的研究成逐年递增, 至 2013 年成下降趋势, 研究越来越专业化, 但同时也呈现出角度单一、研究重复等状况, 故本 文对已有研究进行梳理, 以受众本位的角度为出发点, 分析网络集群行为。

\section{1. 引言}

\section{1 从网络群体性事件出发}

国内对于网络群体性事件的定义中 “网络群体性事件是一定数量的无组织的网民针对某 一共同影响或刺激,在网络环境中或受网络传播影响的群体性努力” [1]在各类期刊中引用较多 且认可度较高。从该定义展开的研究中, 大都集中在定性研究, 着重研究网络群体性事件发 生过程中的群体心理、群体行为, 以及管控方法, 忽略了网络群体形成机制的探索。 
网络群体性事件的基础是网民间的群体互动, 且产生了集群行为。就我国目前的网络环 境而言, 群体互动主要分为公共群体互动 1 与社区群体互动 2 。前者主要体现在某新闻的评论 区、自媒体互动板块（微信朋友圈、微博热搜）等方面, 易导致大型网络群体性事件的产生; 而后者则体现在QQ群、知乎平台、贴吧等, 更易推动网络群体性事件的发生。在网络群体性 事件的研究中, 该互动方式的分类则可作为一个重要的结构化指标, 针对不同类型的互动, 以及产生的不同性质的群体事件进行相关研究探讨。

\section{2 以 “陌生人” 理论为基础进行思考}

个体如何形成群体是一种自上而下的观点，与其相似的自上而下的观念则是如何将形形 色色的个体凝聚成群体以便于控制，提及此观念便离不开帕克的《移民报刊及其控制》。在 将个体凝聚成群体所遇到的阻力, 是否也能类比为个体形成群体过程可能存在的心理, 针对 性的深读《移民报刊及其控制》后, 会发现二者有些共同之处。如果将其作为一个范式, 再 进行网络群体性事件中的个体如何形成群体的研究, 帕克的研究思路可看做另一维度的参考。

那么将 “移民” 和 “网民个体” 做类比是否恰当，二者又有怎样的共通之处？这里就不 得不提齐美尔的 “陌生人” 理论，从简单的概念上理解，网民就是陌生人的一种表现形式。 “这里的陌生人不是此前常常接触过的意义上的外来人, 既不是指今天来、明天走的流浪者, 而是之今天来、明天留下来的漫游者” 3 是齐美尔对陌生人概念进行阐述的一隅。在网络群体 性事件中, 网民正是今天参与到一个事件中, 并且明天进行持续的关注与参与, 从而形成了 网络群体性事件。齐美尔对 “陌生人” 特性描述作用于网民上也可以看做网络群体性事件是 “网民对知情与参与权的需求在网络上可以充分体现”。

\section{2. 试论Web3.0环境下网络群体事件的类别}

Web2.0时代使受众不再是单方面的接收信息，产生了受众间互动，传者和受者的关系更 加融通, 任意受者都可以成为传者, 使信息的传播更加灵活。Web3.0时代则是指网站内的信 息可以直接和其他网站相关信息进行交互; 用户在互联网上拥有自己的数据, 并能在不同网 站上使用。简言之, 则是Web3.0技术使网络信息的整合能力更强, 在任意搜索引擎、网站上 都可以看到全网的信息，使信息的传播不再受到限制; 另外可以使信息传播更具针对性，搜 索引擎、自媒体等会为受众推荐其近期关注的信息，增强受众与信息的密集性接触。

同样，依托于Web3.0技术的信息整合能力，加之自媒体的推广，让整个互联网连成一个 整体。在淘宝客户端搜索了某明星同款鞋子后, 在百度首页就会更多的出现较多该明星的相 关信息; 在网页多次搜索了 “王者荣耀” 后, 可能连续几天的首页信息推送都和王者荣耀有 关。在Web3.0时代，当我们搜索了某方面的信息后，整个网络都会认为我们在关注该信息， 并 “人性化” 的为我们推送相关信息。从网络集体性事件的角度来看, 这种推送方式会使我 们持续关注某方面的信息, 并且会从全网集合信息, 智能挑选关注量较多的推送给我们。以 搜狗引擎为例, 在 “搜狗” 中搜索某一词条, 微信公众号、百度知道、新浪网和知乎论坛等 多个门户网的信息都会出现在网页中, 并且按照点击量、相关度等综合指标按顺序排列。

网络集群行为作为网络群体事件的先导问题，笔者尝试先对网络集群行为进行梳理，在 此视角下探索网络群体事件的类别。

\section{1 情感意识构建网络群体}

理论界对网络集群行为进行明确定义的不多, 有观点认为, 网络集群行为是指一定数量 的、相对无组织的网民针对某一共同影响或刺激, 在网络环境中或受网络传播影响的群体性

\footnotetext{
1 网民所建构和呈现的网络在并不明晰和明确的情况下参与的网络群体的互动。

2 网民在一个相对固定、具有特定议题取向的论坛内参与的网络群体互动。

3 齐美尔著, 林荣远编译: 社会是如何可能的一一齐美尔社会学文选[C]. 桂林: 广西师大出版社, 2002
} 
努力，并基于 “共同关注点、共同信念、共同行动目标” 将网络集群行为分为三类。该观点 论述具有普遍性, 分类也具有代表性, 首次呈现在2013年, 且是主要针对微博进行分析。笔 者将对这以上三类重新建构，从受众心理出发，大致归为五类。

反抗意识 “集合” 的网络群体，该反抗意识主要表现于两种情况，一是对某个现实事件 的抗争, 借助网络表达出来, 并产生共鸣, 组成群体; 二是对某个网络话题有自己不同的看 法, 出于对隐藏的文本的挖掘, 或是个人意识的觉醒, 不愿意从中, 发表个人观点寻求认同。

无意识的网络群体 “融合” ，群体中的每个人并未在主观上有意识的群体，在事件中仅 是自我意识的表达, 且没有明确的诉求。运用这种表达形式的人较多, 或许多个体表达的内 含相同，从而产生了网络群体性事件。

受潜在共性 “吸引”的网络群体某个公共性的事件可能会涉及到自己与自己身边的人, 这类人会由于该 “共性”组成群体。

话题性 “聚集” 的网络群体出于对某个话题喜爱，原本就属于网络中陌生的群体主动的 聚集在一起, 积极的推动某个话题或议题。

现实群体的网络表现形式该类网络群体在现实生活中也属于共同群体，由于客观条件限 制，针对某个事件，为达到既定效果倾向的最大化，在网络上聚集，并进行决策。

\section{2 群众心理诱发群体事件}

由于网络群体性事件的复杂性与灵活性, 很难从做出绝对的结构化分类, 现就事件的相 对性, 以受众群体与事件的关系为切入点, 从受众对事件的关注时间、事件是否具有公共性、 受众间是否存在较多的互动, 以及是否有人进行组织等四个角度分类。针对不同类别的网络 群众性事件，可对群众心理做出分类探讨。

2.2.1 流动性关注群体事件与持续性关注群体事件

从概念上来讲，某微信公众号的文章如果达到100000+，并且引起热议，对受众的态度及 行为造成影响, 在一定层面上已经属于网络群体性事件。其中微信公众号 “咪蒙” 推文《别! 再！劝！我！生！二! 胎！了! 》, 在发出四十分钟以内便有了 $100000+$ 的阅读, 点赞数也达 到了尤为少见的 $100000+$ 。该文章得到了与文章态度一致的受众的推崇、转发, 并且加强了其 既有态度倾向。事件的发酵与高潮在短时间内完成并截止，阅读之后虽会影响自身的观念， 但受众在阅读文章之后并未对文章进行持续性关注。此类网络群体性事件的产生是由流动的 受众共同推动的。

持续性群体事件的主体则更多的倾向于事件，具有一定的复杂性，并且会得到媒体的广 泛关注。以 “成都男司机欧打女司机” 事件为例, 最初被媒体报道为 “男司机下车暴打女司 机”，后成为翻转新闻 “女司机变道惹怒男司机被拖下车打成脑震荡”，一度成为大街小巷 的热议话题。不论是媒体和受众皆对该事件进行持续性的关注与探索，大家会对事件进行探 讨, 由于事件的复杂性, 大家会主观且主动的在一定程度上探索事件的本质。

2.2.2 公共性群体事件与针对性群体事件

国内 “公共” 事件很少独立出现，往往和 “突发性” 关联紧密，独立的公共事件很少受 到人们的关注。也有人认为 “公共性事件” 和 “群体性事件” 概念类似, 仅是 “公共性事件” 为中性的, “群体性事件” 为负面的。4在此, 我们谈及的公共性群体事件, 基于本文论述的 “网络群体性事件” 概念基础上，结合 “公共性” 5本身的概念。如 “小悦悦事件” 可能会在 所有有孩子的人身上重演，而2016年的 “帝吧出征事件”也关乎到中国民众共同的爱国情怀。 公用性网络群体事件的受众覆盖面较广, 容易引起较大的影响。

\footnotetext{
4 熊光清. 《中国网络公共事件的演变逻辑》[J]. 社会科学, 2013，（04）

5 一是关系到公共利益, 或不特定多数人的生命健康和财产安全等; 二是进入大众视野, 引起社会广泛关注。
} 
针对性的网络群体事件覆盖面大小各异，大众对事件的关注具有明显的分界线，但是易 出现极化态度。以 “鹿晗恋爱” 事件6本体为例, 排除各类文案运营, 该事件的参与者绝大部 分属于鹿晗的粉丝, 或相关娱乐内容的受众, 除此之外参与到事件中的受众极少。但是, 一 旦参与到事件中则会展现出狂热、极端或尤为重视的态度。

\subsection{3 强互动性群体事件和个体推动性群体事件}

强互动性的网络群体事件指网民在事件中有较多的互动，该互动主要表现在网民间的相 互评论、讨论以及转发, 如2014年的 “快播事件” ，快播被封、王欣被抓在网络上引起了一 系列的讨论, 并且讨论具有明显的议程, 且议程主要由 “自来水” 7推动。网民分别在议程下 发表意见进行讨论, 并且不断对议程进行改变、拓展, 并且部分的议程会持对立状态, 产生 一定的言语冲突。

而个体推动性的网络群体事件则互动性较弱、或受众间没有主观上的互动，主要体现在 网民依靠自我意识来推动某个话题, 因这类网民的数量较大, 最终将话题推动成为群体性事 件，但在推动过程中网民间的主观互动极弱。如 “十九大” 期间, 微信朋友圈中各种关于十 九大的文章分享, 虽说相互转发别人的文章属于互动, 且会被别人的态度所影响, 但这不是 主动的做互动行为, 评论、讨论等常规的主观互动很少出现。另外, 如网上《国家要在XX省 选出 $\mathrm{X}$ 个重点建设城市, 由你投票决定》等虚假网络新闻, 大家在看到后都会有一个主观的 判断, 但又会点进去投票并且转发。在事件的推动过程中仅仅是单项的传递, 没有对现实生 活产生特定的影响，但是，却会在某方面提升群体的共同认知或集体荣誉。

2.2.4 有组织性的群体事件和自发性的群体事件

该方式的分类标准较为明确, 前者是有组织的、有目的性的网络群体性事件，但区别与 组织传播, 其 “群体” 具有 “网络群体” 的特征, 是松散的、临时组织的、由共同的话题而 集中在一起的。而自发性的群体事件则是不受 “组织者” 所领导, 自发、自愿、自主的参与 到事件中，并由于参与的受众较多，最终共同推动网络群体性事件的产生。

\section{References}

[1] Robert E.Park, The Immigrant Press and Its Control[C].China Renmin University Press,2011

[2] Ao Zheng. The Study on Netizens' Self-conciousness in Network Interactivities [D]. Communication University Of China, vol. 06, 2008.

[3] Ying Huang. Reseaerch on the Coverage of Public Events of Traditional Media--Based on the Typical Report of Public Events from 2007 to 2011 [D] , Shandong University, vol. 04, 2012.

[4] Bo Peng. Network Commmunitive Event Rule Analysis and Guard Strategy Research[D]. Shanghai Jiao Tong University, vol. 06,2008.

[5] Huiming Zhou. The Research of Group Psychology in Network Public Opinion[D]. Guangxi Teachers Education University, vol. 06, 2016.

[6] Mengshi Sun. Researches on How Individual Changes in Group:Take Chinese Army Group as Research Object[D]. Liaoning University, vol. 05, 2012.

[7] Lidan Chen. The Psychological Mechanism of Group Communication $[\mathbf{J}]$. Southeast Communication, vol. 01, 2016.

[8] Jianjuan Yang; Fei Wu. Understand the "Marginal People" Living in Other Places-Talking about Parker's Concern [J]. journalism Research, vol. 05, 2012.

\footnotetext{
6 娱乐明星鹿晗在新浪微博公布恋情，其粉丝大量评论转发，导致新浪微博短时间系统崩溃。

7 即免费水军, 出于对某件事的喜爱或关注, 以自愿为原则而发起宣传、进行推动的行为。
} 